\title{
FEMORAL NECK FRACTURE;
}

FEMORAL NECK FRACTURE TREATED WITH HEMIARTHROPLASTY BY USING AUSTIN MOORE PROTHESIS (AMP)-SURGICAL OUTCOMES

1. MBBS, MS (Ortho) Assistant Professor Department of Orthopedics Allied Hospital, Faisalabad.

2. MBBS, FCPS

Senior Registrar Department of Orthopedics DHQ, Hospital Faisalabad.

3. MBBS, FCPS

Associate Professor

Department of Orthopedics Faisalabad Medical University,

Faisalabad.

Correspondence Address:

Dr. Basharat Manzoor

Address: 50-B, Peoples Colony No.1

Faisalabad.

drbasharat5050@gmail.com

Article received on:

21/11/2017

Accepted for publication:

15/04/2018

Received after proof reading: 03/12/2018

\section{Basharat Manzoor ${ }^{1}$, Shehzad Mehmood ${ }^{2}$, M. Javed Iqbal $^{3}$}

ABSTRACT... Femoral neck fracture is common in the elderly, with only $2-3 \%$ found in patient younger than 50 years. Osteoprosis \& co-mordities increase the incidence of their fractures. Non-Operative treatment is indicated only is unfit or non-ambulatory patients age, general health status and Socio economic condition of patients along with availability of facilities are important to select the treatment modality. There are many operative treatment options, prosthetic replacement with Austin Moore prosthesis (AMP) is one of the treatment option in this age group. Post Operative complications are thigh pain, hip dislocation \& infection. Objectives: To determine the results of Austin moore prosthesis in fracture neck of femur in elderly patients in terms of mid-thigh pain. Study Design: Descriptive Case series \& setting in Orthopedic Department Allied Hospital Faisalabad. Period: Study Period is from April 2008 to August 2010. Setting: All the cases done in Orthopedic department Allied \& DHQ Hospital Faisalabad. Methods: All the patients were evaluated in emergency department of Orthopedics as per ATLS protocol and diagnose by obtaining X-Rays pelvis with both Hip Joints and were operated by consultant through moore's approach Head of Femur was removed and replaced by Austin moor's prosthesis of appropriate size. In all cases one dose of prophylactic broad spectrum antibiotic were giving. Results: 56 Patients (31 males $55.4 \%+25$ female 44.6) with mean age $73.08+8.13$ are Included in the criteria and were operated with same implant. After 2 months post operatively 40 Patients had no pain 07 (12.5\%) had mild (71.4\%). Pain \& 08 Patients 14.3 $\%$ had moderate pain. After 03 month post operatively 43 Patients $(78.2 \%)$ had no pain, 02 (3.6\%) had mil, 08 Patients (14.5\%) moderate and 01(1.8\%) had scene. Conclusion: Prosthetic replacement with AMP is one of the treatment options in femoral neck fracture.

Key words: Austin Moore's, Hemin Arthoplasty, Neck of femur fracture.

Article Citation: Manzoor B, Mehmood S, lqbal MJ. Femoral neck fracture; femoral neck fracture treated with hemiarthroplasty by using austin moore prothesis (AMP)-surgical outcomes. Professional Med J 2018; 25(12):1805-1808. DOI: $10.29309 / \mathrm{TPMJ} / 18.4530$

\section{INTRODUCTION}

In elderly people fracture neck of femur is more common than younger. ${ }^{1,2}$ These fractures comprise about $20 \%$ of work load on an orthopedic trauma unit. $^{3}$ In hip fracture about $50 \%$ fracture of the neck of femur are within the capsule of the hip joint.

Fracture of proximal femur in elderly patients is a challenging problem for orthopedic surgeons all over the world. Female frequently have fracture neck of femur the incidence increases with age of patients. There are numerous risk factors with an increase risk of hip fractures, include less sunlight exposure, decrease in physical activity, smoking, and history of steroid intake. ${ }^{4}$
Treatment of femoral neck fracture is controversial and challenging all over the world. The aim of management of the fractures is to restore the functional outcomes of the patients. Many options for internal fixations with screws, plates and hemi or total hip arthroplasty are available. There is a high rate revision due to non union and a vascular necrosis after open reduction and internal fixation. ${ }^{5}$

In developing countries like Pakistan hemi arthroplasty is perform frequently in elderly patients having femoral neck fractures with shorter life expectancy. Management of with austin moore prosthesis has no chances of non union 
and a vascular necrosis. Other complications like rear failure, bed sores D.V.T \& chest infections still occur. Usually the injuries of the hip fracture are isolated but others fractures like distal radial fractures, humeral fracture and fracture shaft of femur so physical exanimation and x-ray evaluation must be done to rule out these injuries.

\section{Review of Literature}

In an orthopedic \& trauma unit $20 \%$ of the load of operative work is comprised of femoral neck fractures $40-50 \%$ women 30-22 men having high risk of fracture of the hip joint in their life time. Due increasing life expectancy worldwide femoral neck fractures increasing from 1.66 million in 1990 to 6.26 million in $2050 .^{6,7,8}$ This is big burden to health system to treat fractures annually.

Patients less than 60 years old have less chances of fracture of the neck of the femur than elderly patients especially female. Due to racial variation black races have less incidence than white and female are involved more than male..$^{9,10,11}$ In North America and white population in Europe having more incidence these fracture within 2 years . $5 \%$ in male and $10 \%$ in females having high risk of 2nd fractures of the hip joint. ${ }^{12,1314}$

The incidence of these fracture increase worldwide till 2050. In Europe studies showed osteoprotic fractures are going to be reduced..$^{15,16}$ In other study the incidence of femoral hip fracture will reduced in number. It is not clear that these changes in reduction in numbers are due to therapeutic or preventive modalities. The chances of fracture in osteomalicia, renal osteodistrophy and metabolic disorders have high risk of femoral neck fractures.

\section{METHOD \& MATERIAL}

All patients were selected according to diagnostic criteria admitted through emergency department and out patient department. All these patient were evaluated for life threatening condition in emergency department as per ATLS protocol. Diagnosis was made by obtaining $X$-Rays pelvis with both hip joint. Patients were kept on skeletal traction till the definitive surgery. After all base line investigations, ECG, Chest x-rays to rule out any other systemic comorbid disease. Patients were prepared for spine position and were operated by consultant orthopedic surgery through posterior approach by placing patient in lateral position (Moor's approach) the head of femur were removed \& will be he replaced by austin moor prosthesis of appropriate size in all cases single dose of prophylactic broad spectrum antibiotic were given and were continued post operatively. Patients were mobilized on next post operative day \& physiotherapy was started skin stitches were removed on $12^{\text {th }}$ post operative day and farther follow up at two weeks \& 3 months.

\section{RESULTS}

There were a total of 56 cases falling in the including criteria. These were operated with bane implant with AMP mean age was 73.08 \pm 8.13 . Youngest patients was 65 year old with oldest one was 95 years of age. There were 31 (55.4\%) male and 25 (44.6) female.

Femoral neck fracture encountered in this study were described according to their respective garden classification.

Results obtained is terms of type of fracture were as following.

Garden Type I were 3 (5.4\%)

Garden Type II were 8 (14.\%)

Garden Type III were 34 (60.7\%)

Garden Type IV were 11 (19.6\%)

Out come of the patients was, broad upset post operative mid thigh pain at 2 weeks and 3 months results obtained were as followings:

Post operative pain explicit in front and mid thigh scored on a 4 pint ordinal scale. (No pain, Mild, Moderate, Severe). Pain will be assessed on numeric pain intensity scale. In this scale patients is instructed to rate their pain.

1- ASK patients to rate his pain on scale from zero to ten.

2- Zero mean have no pain at all

3- Ten means the worst possible pain patients can imagine.

4- These values on pain scale will be allocated as. $0=$ No pain 
1-3 = mild pain

4-6 Moderate Pain

7-10 $=$ Severe pain

Mid Thigh Pain at 2 Weeks

Patients who had no pain 40 (71.4\%)

Patients who had mild pain 07 (12.5\%)

Patients who had moderate pain 08 (14.3\%)

\section{Mid Thigh Pain at 3 Months}

Patients who had no pain 43 (78.2\%)

Patients who had mild pain 02 (3.6\%)

Patients who had moderate pain 08 (14.5\%)

Patients who had Severe pain 01 (1.8\%)

02 patients died during follow up so mortality up

to 13 months was $0-(3.6 \%)$

\section{DISCUSSION}

Femoral Neck fracture are one of the commonest fracture in elderly people, with only 2-3 found in patients younger this 50 year 17 years of age.

The patients have to return to pre fractures functional state after the treatment is the goal of the management. Early mobilization is necessary to avoid the problems associated with recumbency like DVT, bed sour, respiratory tract infection.

In younger patients, treatment of fractures is closed reductions and internal fixations in older patients internal fixation options with screws, plates and hemi arthroplasty or THR. ${ }^{17,18}$ There is a high incidence of non union, a vascular necrosis and revision surgery after open reduction and internal fixation method.

Treatment of the older patients depends on patient's profiles local condition, trainings and preference of surgeon. Patients older than 65 years are mostly treated with some form of prosthetic replacement. ${ }^{19}$ Options for with (Prosthetic replacement include hemi arthroplasty (Uni polar \& Bi Polar) and total hip arthroplasty.

In elderly patients over 65 year hemi arthroplasties with AMP is a good choice of procedure in fracture of neck of femur. After this procedure patients return pre morbid level of activity occur very quickly without complication of prolong in mobility.

The present study has evaluated the results of austin moor prosthesis replacement in terms of an important complication of this procedure i.e. post operative mid thigh pain. 56 patients were included in the study out of which 31 (55.4\%) was male and $25(44.6 \%)$ were female mean age was $73.08 \pm 8.13$.

Ozwrkmeny et al 120 compared the results of cemented verses non cemented hemi arthroplasty in 48 patients including 29 (60.4\%) females and $19,39.6$ person in males. Mean age was 88 year. Frihagen et al compared the Hemi arthroplasty and internal fixation results in 222 patients out of which 165 were females $72 \%$.

Alnal et al conducted a study to determine the results of $\mathrm{HA}$ in terms of mortality and morbidity in 46 patients with average age of 70 years. Male $\&$ female ration $1: 2$.

In present study post operative mid thigh pain was evaluated at 2 weeks and 3 months postoperatively At $\mathrm{HO}$ and of 2 weeks most of the patients $(71.4 \%$ no pain, while $12.55 \%$ had mild pain and $14.3 \%$ had moderate pain.

At The end of 03 months, most of patients $78.2 \%$ had no pain, while $1.8 \%$ had severe pain, $14.5 \%$ had moderate and $3.6 \%$ had mild pain.

OZ turkmen y et al study shows 48 patients treated with hemi arthroplasty observed 34 patients (71.\%) with no thigh pain while 14 patients (29\%) with thigh pain of variable intensity i.e mild, moderate or severe.

Ahmed I et al observed $17.4 \%$ patients with thigh pain of variable intensity. Narsish AR et al treated 500 patients with AMP, result was $81 \%$ with no pain or minimal pain, while 6 person had constantly pain in hip joint.

In present study, mortality rate up to 2 weeks $1.8 \%$ and was $3.6 \%$ at 3 months post operatively. Ahmed I et al. found that mortality at 2 weeks was $4.3 \%$ and $26 . \%$ at one year and was high 
among the patients who had associated systemic commodities. Management of fractures neck of femur his always been controversial and debatable due to variable experience by various institutes and availability of treatment modalities.

\section{CONCLUSION}

Femoral neck fracture is a common fracture among the elderly people and commonly follows trivial trauma at home. The aim of the management of the patients is to take him or her at pre fractures state of health. In elderly patients who need to be mobilized as easily as possible to prevent the complications of prolonged immobilization, prosthetic replacement with AMP is an appropriate mode of treatment.

\section{Copyright(C) 15 Apr, 2018.}

\section{REFERENCES}

1. Berglud Roden $M$, Swierstra BA, Wingstrand $H$, Thorgren KG. Prospective Comparison of hip fracture treatment 856 cases followed for 4 months in the nether lands. And swedan, Acta orthop scand 1994; 65:287-94.

2. Damany DS, Parks MJ, Chojnouiski a complication after intra capsular hip fracture in young adults. A meta analysis of 18 published studies involving 564, fractures injury. 2005; 36:131-41.

3. Singer BR, MeLauchlan GJ. Robinson CM, et al Epidemiology of fractures in adults: the influence of age and gender. J Bone Joint Surg Br 1998:80:243248.

4. Dennison E, Muhammad MA, Coopere, Epidemiology of osteoporosis, Rheum dis clin north Am 2006; 32:617-629.

5. Marya SKS, Thukral R, Singh e. Prosthetic replacement in femoral neck fracture in the elderly: Results and revies of the literature, indication J orthop 2008; 42:617.

6. Davinson JN, Calder ST, Andreson GH, Ward G,
Jaggerc, Harper wm, et al. Treatment for displaced intra capsular fracture of proximal femur. A retro respective randomized trial in patients $65-79$ years. $J$ Bone Joint Surg for 2001; 83:206-12.

7. Rog markc, Carlsson A, Johnell O, sernbol. A prospective randomized trial of internal fixation versus arthroplasty for displaced fracture on neck of femur. Functional out come for $\mathbf{4 5 0}$ patients at two year. J bone joint surg Br. 2002; 84:183-8.

8. Glyn AP. Fracture of hip surgery. 200; 49: 105-9.

9. Eyichvkwu Go, Lyidobi EC. Austim-Moore hemi arthroplasty; The enugu experience niger. $\mathrm{J}$ Med 2007; 16:125-8.

10. Eiffors $L$ Gull berg B Alexander $E$ et al. Methodology of medos-multicenter; Validity and relevance consideration bone. 1993; 14: S 45-49.

11. Chevalery T, Guilley E, Herrmann FR,et al. Incidence of hip fracture over a 10 year period (1991-200): Reversal of a secular trend. Bone 2007; 40:1284-1289.

12. Fukushim $T$, Sudo $A$, vehida $A$. Bi Lateral hip fracture. J orthop sci 2006; 11:435-38.

13. Rock wood Book.

14. Rock wood Book.

15. Robinson CM, Saran D, Annan IH. Intra capsular hip fractures. Results management a do patients in a treatment protocol. Chin orthop Relat Res 1994; 83-91.

16. Piirtola $M$, Vah/berg $T$, I soaho $R$, et al. Incidence of fracture and changes. Over time among the aged in a finish miomicipality: A population based 12 year follow up aging clin express. 2007; 19: 269-276.

17. Kannus P, Parkkari J, Sievanen H, et al. Epidemiology of hip fractures. Bone 1996; 18:57-63.

18. Chalmers J, Irvine GB. Fracture of the femoral head in elderly patients. Clin orthop 1988; 229:125-130.

19. Chalmers J, Irvine GB. Fractures of the femoral head in elderly patients. clin orhtop 1988; 229:125-130.

\section{AUTHORSHIP AND CONTRIBUTION DECLARATION}

\begin{tabular}{|c|l|l|r|}
\hline Sr. \# & \multicolumn{1}{|c|}{ Author-s Full Name } & \multicolumn{1}{|c|}{ Contribution to the paper } & Author=s Signature \\
\hline 1 & Basharat Manzoor & Author Data analysis & Shatrzad \\
2 & Shehzad Mehmood & Data collection & \\
3 & M. Javed lqbal & Final review & \\
\hline
\end{tabular}

